\title{
BMJ Open Early childhood constraint therapy for sensory/motor impairment in cerebral palsy: a randomised clinical trial protocol
}

\author{
Olena Chorna, ${ }^{1}$ Jill Heathcock, ${ }^{2}$ Alexandra Key, ${ }^{3}$ Garey Noritz, ${ }^{4}$ Helen Carey, ${ }^{1}$ \\ Ellyn Hamm, ${ }^{1}$ Mary Ann Nelin, ${ }^{1}$ Micah Murray, ${ }^{5}$ Amy Needham, ${ }^{6}$ \\ James C Slaughter, ${ }^{7}$ Nathalie L Maitre ${ }^{1,3,4}$
}

To cite: Chorna 0 ,

Heathcock J, Key A, et al. Early childhood constraint therapy for sensory/motor impairment in cerebral palsy: a randomised clinical trial protocol. BMJ Open 2015;5: e010212. doi:10.1136/ bmjopen-2015-010212

- Prepublication history for this paper is available online. To view these files please visit the journal online (http://dx.doi.org/10.1136/ bmjopen-2015-010212).

Received 7 October 2015 Revised 13 October 2015 Accepted 14 October 2015

CrossMark

For numbered affiliations see end of article.

\section{Correspondence to}

Dr Nathalie L Maitre; Nathalie. maitre@nationwidechildrens. org

\section{ABSTRACT}

Introduction: Cerebral palsy (CP) is the most common physical disability in childhood. It is a disorder resulting from sensory and motor impairments due to perinatal brain injury, with lifetime consequences that range from poor adaptive and social function to communication and emotional disturbances. Infants with CP have a fundamental disadvantage in recovering motor function: they do not receive accurate sensory feedback from their movements, leading to developmental disregard. Constraint-induced movement therapy (CIMT) is one of the few effective neurorehabilitative strategies shown to improve upper extremity motor function in adults and older children with $\mathrm{CP}$, potentially overcoming developmental disregard.

Methods and analysis: This study is a randomised controlled trial of children 12-24 months corrected age studying the effectiveness of CIMT combined with motor and sensory-motor interventions. The study population will comprise 72 children with CP and 144 typically developing children for a total of $\mathrm{N}=216$ children. All children with $\mathrm{CP}$, regardless of group allocation will continue with their standard of care occupational and physical therapy throughout the study. The research material collected will be in the form of data from high-density array event-related potential scan, standardised assessment scores and motion analysis scores.

Ethics and dissemination: The study protocol was approved by the Institutional Review Board. The findings of the trial will be disseminated through peerreviewed journals and scientific conferences.

Trial registration number: NCT02567630.

\section{INTRODUCTION}

\section{Background and rationale}

Cerebral palsy (CP) is the most common physical disability in childhood. ${ }^{1}$ It affects 3.6/100 children in the $\mathrm{USA}^{2}$ with $\sim 10000$ new diagnoses every year. ${ }^{3}$ The prevalence of
$\mathrm{CP}$ in developing countries is estimated to be $\sim 5-10$ times greater. ${ }^{4} \mathrm{CP}$ is a disorder resulting from sensory and motor impairments due to perinatal brain injury, ${ }^{5-8}$ with lifetime consequences that range from poor adaptive and social function to communication and emotional disturbances, ${ }^{9}$ all contributing to a shortened life expectancy. ${ }^{10-14}$ The societal costs are difficult to estimate but the financial burden is well over $\$ 1 \mathrm{M}$ per life affected. ${ }^{15-17}$ A growing number of evidencebased therapies aim to improve gross motor function through changes in body structures and function in children with CP (eg, hip surveillance, surgery). ${ }^{18}$ However, infants with $\mathrm{CP}$ have a fundamental disadvantage in recovering motor function: they do not receive accurate sensory feedback from their movements, ${ }^{19} 20$ leading to neglect of an affected extremity and difficulty learning new movements, a process called developmental disregard (DD). ${ }^{21-23}$ As a consequence, even children who receive time-intensive and resource-intensive standard therapies have stable or declining motor function and developmental trajectories that do not 'catch up' to those of typically developing (TD) children. ${ }^{24-28}$ DD can then lead to school-age learning problems, decreased participation in physical and social activities and costly long-term mental and physical morbidities. ${ }^{29-32}$

Constraint-induced movement therapy (CIMT) is one of the few effective neurorehabilitative strategies shown to improve upper extremity motor function in adults and older children with $\mathrm{CP},{ }^{33-35}$ potentially overcoming DD. It is mainly applicable to CP patients who are diagnosed with asymmetric or hemiparetic forms of the disorder, ${ }^{36}$ in which one side of the body is more affected than the 
other. CIMT is based on the premise that preferential use of an affected upper extremity (by constraining the less affected one), and shaping with repetition of movement by skilled therapists, can overcome neglect and restore function of that extremity. ${ }^{22}$ However, published pediatric CIMT studies have multiple limitations:

Participants are mostly older children past the period of maximal neural plasticity in early childhood ( $\sim 3$ years of age) when interventions have the potential for greatest impact. ${ }^{37} 38$

Developmental trajectories are rarely examined, mostly due to the challenges of intensive, specialised therapy and limited options for objective assessments in very young and often in preverbal patients.

Large randomised controlled trials (RCTs) are rare in the USA ${ }^{39-43}$ This may be due to the difficulties of equipoise in this vulnerable population, where parents believe that any treatment is better than none ${ }^{44-46}$ and studies indicate benefit without harm.

Therapeutic strategies focus on development of new motor patterns and pathways and rarely account for the importance of connectivity between sensory and motor systems in inferring new movements.

Our protocol uses a multidisciplinary approach to overcome these challenges and leverages the established experience with CIMT, infant motor learning of reach, and novel objective measures of sensory function, all unique to our research group. We plan an RCT to test the effectiveness of CIMT, combined with motor and sensory-motor interventions in children aged 12-24 months, using objective, quantitative measures to assess somatosensory, kinematics and developmental motor function. The goal of this project is to provide both clinical data on the value of CIMT in infants and mechanistic data to inform improved designs of interventions for $\mathrm{CP}$ in infancy.

Our intervention and assessment methodologies allow a trial in children aged 12-24 months at intervention start, when neural plasticity and potential downstream benefits are optimal. ${ }^{474}$

By combining standard motor assessments and biomechanics of reach with cortical measures of sensory function, we address mechanistic gaps in the role of somatosensory processing in motor impairment in CP.

Our quantitative measures of the treatment's ability to change sensory processing and upper extremity function will inform future CIMT design modifications.

Use of a wait-list control for comparison to the intervention allows every infant with CP to eventually receive CIMT. We study timing of CIMT only within the context of developmental trajectories, not in the RCT, to avoid a common problem of confounding amount of total treatment with age at treatment entry.

We examine the role of CIMT on the developmental trajectories of children with CP referenced to TD children and the role of severity of CP in treatment response.

From a public health perspective, our use of a low-cost, low technology version of CIMT with a proven parent-driven training component allows adaptation of the therapy to a socioeconomically and geographically diverse population.

Our novel approach to therapy design for paediatric $\mathrm{CP}$ addresses 'the gaps in the continuum of translational research and the WHO-ICF framework' in order to 'increase the clinical and societal relevance of rehabilitation research throughout the NIH and is thus consistent with recommendations by the Blue Ribbon Panel on Rehabilitation Research' (NIH, June 2012).

To accomplish the goals of our protocol and meet the mandates of the National Center for Medical Rehabilitation Research, our focus, methodology and design are creative and highly novel. The only NIH-funded trial of CIMT in infants aged 6-18 months ${ }^{49}$ tests intensive therapist-assisted training in the home $(3 \mathrm{~h} /$ day) and placement of a non-removable cast versus a splint or no constraint. In addition to concerns of immobilisation of the unaffected extremity, that project does not study the impact of motor learning on developmental trajectories and reach kinematics, and does not address the mechanistic implications of cortical sensory function in CP.

Study the effects of rehabilitation in children aged below 2 years: To date, the studies that have focused on this population included a small number of participants, due to the challenges mentioned above. ${ }^{39}$ We address the logistical issues of enrolling very young children as we have done before ${ }^{50-52}$ by recruiting participants in our large neonatal intensive care unit (NICU) follow-up programmes, where we evaluate infants systematically from birth to 3 years. Former NICU patients constitute $>60 \%$ of all children with CP due to consequences of prematurity or perinatal insults. ${ }^{53}$

We use novel infant-friendly methodologies to quantify cortical sensory function. Our event-related potential (ERP) assessment is rapid, non-invasive and provides quantitative temporal information on neural activation in response to a non-noxious sensory stimulus. ${ }^{54}$ This is an important advance from prior studies using electrical stimulation $^{55}$ or lengthy neuroimaging protocols, that are poorly tolerated by infants and toddlers or require sedation. ${ }^{55-58}$ It also overcomes the feasibility and reproducibility issues in conventional behavioural assessments of sensory function in infants noted by specialists in the field. ${ }^{50}$ Our methodology requires neither active participation nor directed attention. As opposed to behavioural measures of sensory function requiring communication or complex cognitive functions, we can therefore obtain quantitative and relevant information on the group response to treatment to help optimise future intervention designs. Our new analysis techniques can also assess the integrity of responses for children at the individual level.

Focus on improving DD: While adult rehabilitation after stroke concentrates on overcoming neglect or lack of sensory awareness, ${ }^{61}{ }^{62}$ paediatric rehabilitation needs to address the problem in the context of a developing 
human instead of a mature one. ${ }^{63}$ In children, the consequences of the original lesion are amplified ${ }^{21} 6566$ and entire classes of behaviours never develop. Our approach tests the immediate effectiveness of CIMT and also examines it in the context of developmental trajectories.

\section{Design}

We are conducting a randomised trial with wait-list control design of CIMT in children with CP using a waitlist control group. This design allows every child with CP to eventually receive the treatment and avoids issues of equipoise. The RCT portion of the study extends only for a 7-month period, from baseline to 6 months after the 1-month CIMT ends. For CP wait-listed controls, the study continues for 6 months following the CMIT intervention, separate from the completed RCT. We will reference data from both groups to a cohort of TD children to determine developmental trajectories.

Rationale for our design: Because CIMT has been proven to be beneficial in adults and older children, and does not appear to result in any lasting harm, ${ }^{36}$ parents and medical personnel believe that receiving the intervention is better than not receiving it and thus lack equipoise. In our studies and those of others in the USA, consent to participate in paediatric CIMT studies approaches $100 \%,{ }^{39-43}$ with long waiting lists of interested participants. We know from our experience and clinic demographics, that we can expect $\sim 6$ patients with newly diagnosed CP in the requisite age group per 3-month period, making possible a design of staggered enrolment of control and intervention groups in an RCT.

\section{Design implementation}

Every 3 months, six children who meet inclusion criteria are randomly assigned to the intervention or control group (figure 1). All children continue routine therapy. The six children in the intervention group also receive the CIMT intervention for 1 month. For this group, the daily home programme replaces their standard-of-care home programme. All children complete baseline, 1-month and 7-month assessments. After the 7-month assessment, the intervention group has completed the study and resumes routine care. After the 7-month assessment, the control group receives CIMT for 1 month and is assessed 6 months later $(7$ months after the start of CIMT, as in the intervention group), completing the study.

TD children matched for gestational age, gender and corrected age (CA) are tested at baseline or 7-month time points-to reference changes in the CP sample to TD-mean performance.

We have addressed problems of RCT design ${ }^{67}$ as follows:

Randomisation: To strengthen interval validity of the design, randomisation will be carried out using unified reproducible methods (ie, saved random number seeds) provided by the Biostatistics core. We will use a permuted block randomisation scheme with random block size and stratified by age at CP diagnosis. Data analysts will be blinded to group assignment. Intervention fidelity: Checks on the implementation of the home-based intervention are built into the design (see below) and between-group differences in children with CP are clearly delineated during the RCT period of months $0-7$, as controls will only receive standard of care protocols as directed by their paediatric occupational and physical therapists. Monitoring of constraint wear time is built into the constraint.

Differential attrition: Some participants may not return for all visits or complete all assessments and hence may have missing values for response variables. Often, such dropouts are not a random sample of the entire cohort, and analysis of only the complete cases would bias the results. ${ }^{68}{ }^{69}$ We will obtain the specific reason for dropout on a case-by-case basis. Indirect information about non-random dropouts will be obtained by using binary logistic models to predict the
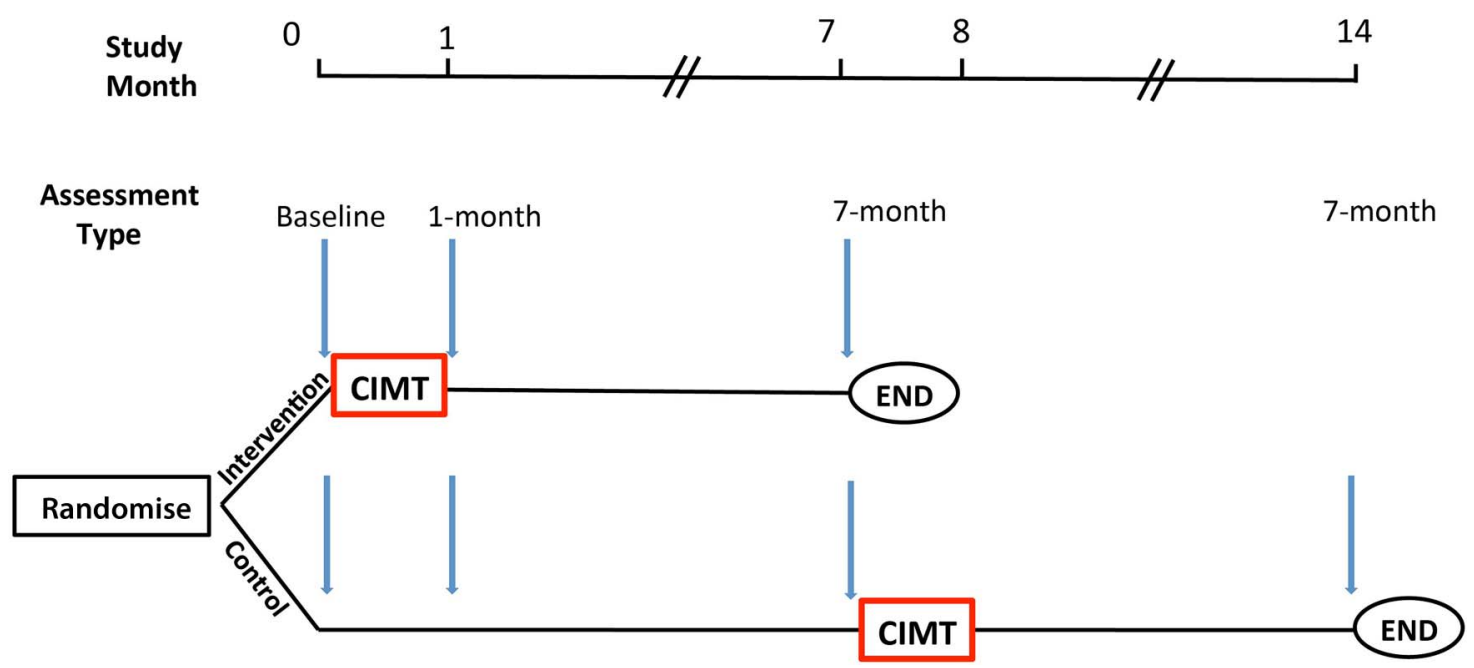

Figure 1 Randomised controlled trial with wait-list control design. (CIMT, constraint-induced movement therapy). 
dropout probability using baseline variables. Impact of dropout on our results will be compared using assumptions that vary from missing at random to informative missing.

\section{Study population}

Inclusion and exclusion criteria

The study population will comprise 216 children, 72 with CP and 144 with TD. We anticipate the distribution to be $60 \%$ male and $40 \%$ female, as CP affects males more frequently than females and represents the distribution at our institution, ${ }^{50} 70$ and we will match TD children for sex and gestational age. All races and ethnicities will be included, with an expected skew towards slightly more African American children than in the general population as described in the enrolment table. Because we will include a large proportion of former preterm infants, we will account for GA at birth (GA) as a marker of immaturity and for CA at assessment, the age of the child from the expected date of delivery, as a representation of maturational age. CA is calculated by subtracting the number of weeks born before 40 weeks of gestation from the chronological age, therefore for term infants, CA is identical to chronological age. ${ }^{71}$

$C P$ children ( $n=72$ ): Inclusion criteria will be diagnosis of hemiparetic or asymmetric CP as determined by published algorithms ${ }^{72}$ and neurological examinatiom, ${ }^{73}$ with $\mathrm{CA}$ between 12 and 24 months. Exclusion criteria will be CP with Gross Motor Function Classification Score $\left(\right.$ GMFCS) ${ }^{74}$ of $4-5$, corresponding to poor or absent mobility and neck control; receipt of Botox to the affected extremity within 3 months of study entry; or scores of $<70$ on the Bayley Scales of Infant Development (Bayley III) cognitive composite ${ }^{75}$

TD children ( $n=144)$ : Inclusion criteria will be GA and sex-matched compared to the $\mathrm{CP}$ group, with $\mathrm{CA}$ at assessment matched to the CP group at baseline (72 children) and at the 7-month assessment (72 children). Exclusion criteria will be any motor or sensory impairment as defined by neurological examination and/or scaled motor scores below 8 for CA on the Bayley III and cognitive impairment or delays as described for the CP group.

Recruitment of participants:

Institutional Review Board (IRB) approval: This study has been approved by the Institutional Review Board (\#1500547). It was assigned a risk level $1-$ no greater than minimal risk on the Code of Federal Regulations (CFR), (45 CFR 46.404; 21 CFR 50.51). This study will include one site, the Nationwide Children's Hospital in Columbus, Ohio USA.

Recruitment: Clinic staff and/or research coordinator will identify prospective participants during NICU follow-up and Pediatric Rehabilitation visits. The study coordinator, the research assistant, or the principal investigator, who directs the follow-up clinic, will obtain informed consent.
Feasibility: The NICU follow-up clinic sees a yearly average of $58 \pm 5$ children with new diagnoses of asymmetric CP before 24 months CA. New diagnoses in the Pediatric Rehabilitation clinic (not referred by the NICU clinic) average $15 \pm 3$. Thus, $>33$ children with CP are eligible per year.

\section{Intervention}

Rationale for specific CIMT design: CIMT is based on the principle of forced-use of a more neglected upper extremity, as a constraint is placed on the less affected extremity. CIMT interventions in published studies vary widely in the duration and intensity of therapy, but most consider 4 weeks with $2-5 \mathrm{~h}$ of daily motor training as an intense course without adverse effects on the constrained extremity. ${ }^{22} 4276$ Furthermore, an RCT of a standard daily home therapy programme without CIMT of 4 weeks duration showed measurable effects and good parent compliance. ${ }^{77}$

However, CP affects motor pathways in the brain and also affects sensory pathways, which complicates the design of CIMT. In addition to injuries to corticospinal pathways directly controlling movement, neuroimaging studies have also confirmed that the thalamocortical fibres to the sensory cortex contribute greatly to the dysfunction observed in CP. ${ }^{78-81}$ Older children and adults with CP have tactile processing deficits in detection of touch (also called registration or sensation) ${ }^{596082}$ and in perception of tactile stimuli, a more complex neural process that involves interpretation of the sensory information. ${ }^{83}$ Principles of new movement inference established in adult populations show that sensory feedback is essential to the development of effective movement. $^{84-86}$ Imaging and movement studies suggest the same is true of children with $\mathrm{CP}^{87-91}$ making the sensory component of learning essential to overcoming DD (see figure 2). Both extremities must receive sensory input and motor experiences during constraint programmes to prevent possible negative effects on sensorimotor cortex development during a critical phase of brain development. $^{92} 93$

Shaping of movement through guided repetition during CIMT creates experience of successful movement essential to inferring more efficient actions. Increased and higher quality sensory feedback during movement is also crucial to improving the brain's ability to infer new movements (figure 3). Providing this type of training is challenging in children with CP who have limited voluntary exposure to tactile input in their affected extremity. ${ }^{61}$ Furthermore, in infants and young children, action-outcome relations are an essential component of learning new motor skills and self-produced (instead of passive) action is a highly specific requirement of paediatric learning. For this reason, shaping of movement in our population needs to be an active task leading to a successful outcome. Finally, to promote optimal upper extremity function, corticospinal tract and sensorimotor development, a complementary emphasis on bimanual 


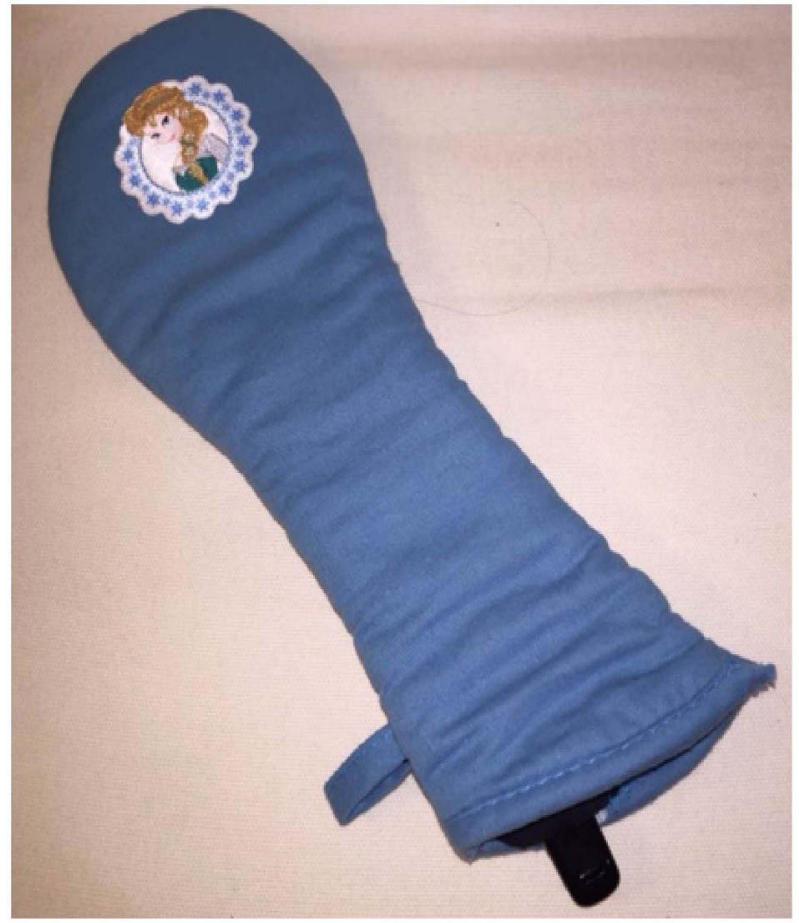

Figure 2 Soft mitt constraint.

play in addition to constraint appears to be of most benefit. $^{95-98}$

\section{Intervention design}

The CIMT intervention includes three components: (1) placement of a removable soft constraint with wearmonitor, (2) demonstration and prescribed home-use of a sensory-motor kit, and (3) a reach/grasp tool (figure 4).

The intervention lasts 4 weeks, with weekly checks during already-scheduled therapy times or other clinic visits. The study therapist will monitor the function of the constrained extremity, download the wear-monitor information, monitor home treatment fidelity and facilitate training.

Constraint: We modified an existing protocol $^{99}$ to suit our population and study focus. We consulted Dr AC
Eliasson regarding the design of a soft long mitt on the less affected arm in the baby-CIMT trial. ${ }^{99}$ In our study, the cast is made from a quilted oven mitt extending from the axilla over the fingers, with a wear-monitor completely covered within a pocket at the top of the mitt (figure 2). The top of the mitt is fastened to fit by 2 Velcro closures. It is worn from infant wake time in the morning for $6 \mathrm{~h}$, or approximately lunchtime. Importantly, during non-training time, caregivers are encouraged to actively facilitate any actions with the non-constrained hand to minimise infant frustration.

Motor-sensory training: As detailed in the rationale, sensory feedback during movements is essential for accurate inference of new movement. ${ }^{100}$ To address this component of learning, we use a sensory kit in which children reach for toys. The kit is used daily for $15 \mathrm{~min}$ and is modified from our previous three CIMT interventions performed in a clinic setting with therapists and non-medically trained volunteers. ${ }^{70} 101$ In the clinic setting, $150 \mathrm{~min}$ of total sensory kit use over 5 days resulted in improvements of grip strength still present 6 months post-treatment. The kit includes bins with age-appropriate toys (chosen for palmar grasp holding) hidden in textured mixes. Parents demonstrate placing hands in the bins and finding toys, then hold children's hands with theirs in the bins, and as children's reach develops, encourage them to reach for partially hidden toys, then fully buried toys. Parents praise children for placing their hands in the bins, regardless of outcome.

Reach training: Because prior experience of successful movement is critical to achieving improved inference of new movements, we modified training to accommodate 12-24-month-old children with CP, based on validated infant-appropriate procedures in which the reaching task is facilitated by using specialised mittens. ${ }^{102}$ These 'sticky' mittens allow infants with immature grasp (comparable to the poor grasp in the affected extremity of children with $C P$ ) to successfully reach and obtain an object from the tray pedestal (figure 5). Studies in the Needham laboratory show that typical 3-month-old infants, who do not have reaching abilities, trained for $10 \mathrm{~min}$ per day in an active reaching task by their parents $(2 \mathrm{~h}$ total

Figure 3 Developmental disregard impairs learning of new and effective movements in children with CP. (CIMT, constraint-induced movement therapy; $C P$, cerebral palsy).

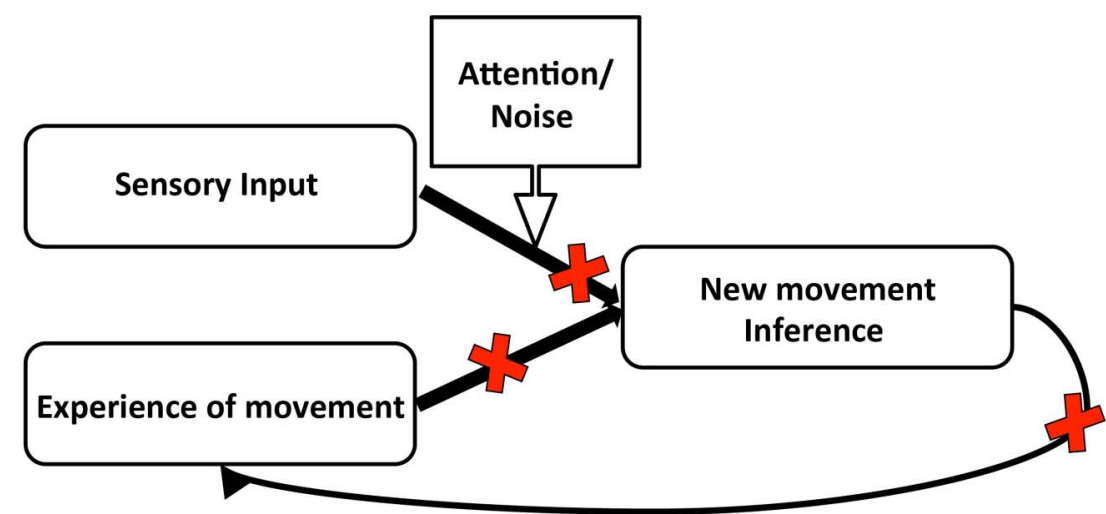




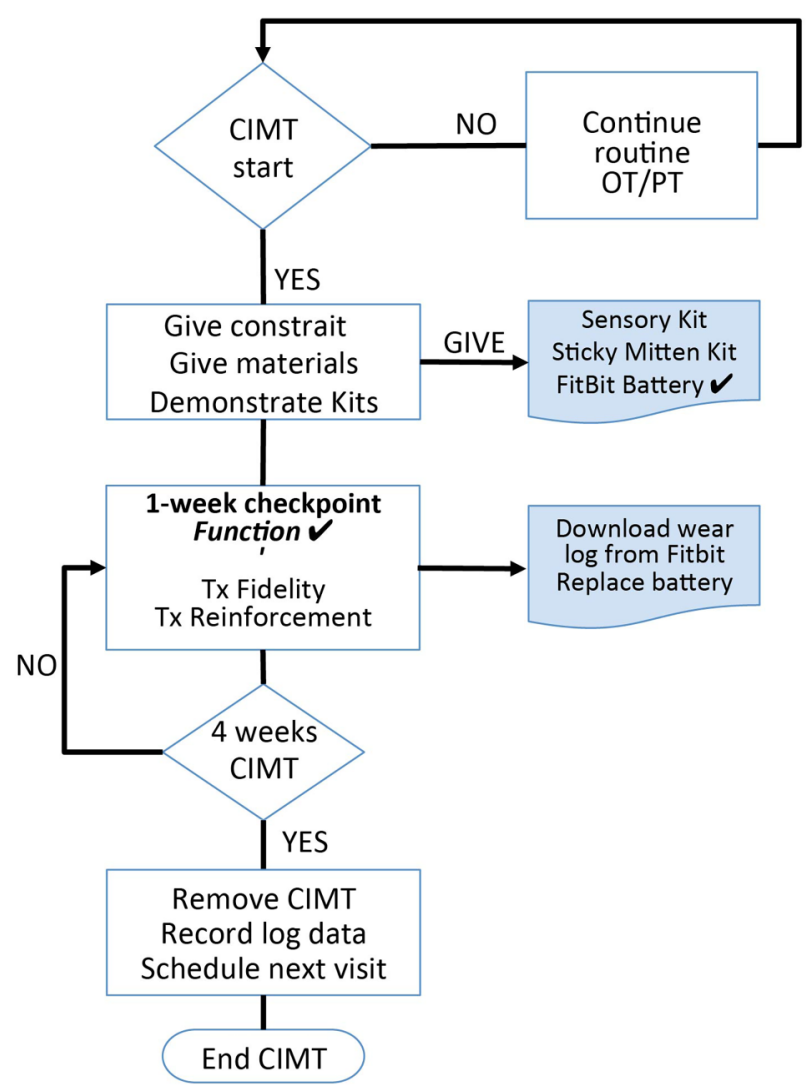

Figure 4 Intervention flow diagram. (OT, Occupational therapy; PT, Physical therapy).

training over 2 weeks) have significant improvements in their reach and grasp behaviours relative to a control group without the mittens. The Needham group further demonstrated that this was due to success during active learning of movement, as passive teaching by observing parents did not improve reaching to the same extent. ${ }^{102-105}$

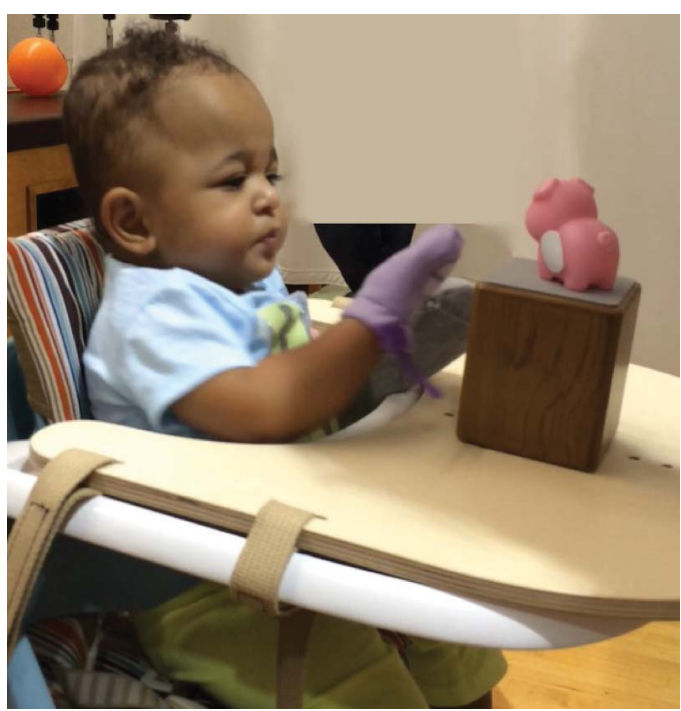

Figure 5 Infant reaching using affected hand with purple sticky mitten (Velcro on palm) for and object with Velcro (pink pig, white Velcro).
Our CIMT intervention lasts 28 days, resulting in $\sim 4.5$ active training-hours of the more affected extremity. Infants receive a custom-made mitten with Velcro (loop side) sewn to the palm, and three sets of training toys, all made 'sticky' with Velcro (hooks side) on one side only. Toys are placed on a (provided) non-movable tray with a pedestal (Zukun Plan, LLC, Columbus Ohio, USA) at full reaching distance on in front of the infant sitting in a high chair. Parents then demonstrate that toys will stick to the child's mitten, place toys back, and draw attention to toys. Parents may move the child's arm to the toy if the infant does not initiate reach, once for each set of toys. Children then must reach out for toys themselves and may manipulate toys before the sequence is repeated for the next toy for a total of $10 \mathrm{~min}$. To ensure good postural support during reach, especially of infants GMFCS of III, infants will be evaluated at intervention start to assess the need for a possible trunk support strap to be added to the high chair.

Support of bimanual activities during non-constrained time: Infants will spend approximately $1 / 2$ their day in the soft restraint and the other half with both hands free. During this time, parents will encouraged to play with a set of provided simple toys and games shown to promote bimanual function and adapted from older children's RCTs. ${ }^{106}$

\section{Monitoring of treatment fidelity}

Duration: The constraint wear time will be monitored using a Fitbit ${ }^{\circledR}$ device incorporated into the mitt. Wear of the mitt activates the Fitbit because the constrained arm still moves either in involuntary small movements or as an assist. When the mitt was on for training and daily living activities, the Fitbit recorded the times as 'awake' or 'restless' with $100 \%$ accuracy for wear time at the minute level.

Frequency of training: Parents will record how many 10 min reaching and 15 min sensory exposure sessions they conducted in a daily paper or electronic log. Alternatively, parents will have the option of documenting task completion through SMS responses to the coordinator's phone, a model currently used to respond to scheduled appointments at our institution. Families will also be provided with support systems, including a therapist (pager and email contact) and a website where they can watch a video of the training.

Quality of training: At the weekly checkpoints, the study therapist will observe parents demonstrate both training tasks in their entirety with the child. During this observation, the therapist will record duration of training, parent implementation of task and parent support of child, using a checklist to compare to the initial demonstration.

The effectiveness of the protective and educational support measures will be assessed on a weekly basis during the intervention and at every assessment time point by the study therapists. Any and all adverse events will immediately be documented and the IRB will be notified according to approved protocols. Participants can withdraw from the study at any time by notifying the 
study coordinator. In the event of adverse effects requiring medical or professional intervention the participants will immediately be cared for by the paediatric providers at our facility at no cost to them.

The hypothetical risks of placing a constraint for $6 \mathrm{~h}$ each day on the less affected extremity will be addressed as follows: prior to encouraging the continued wear of the long mitt each week, both the more and the less affected extremity will be tested for grip and pinch using dynamometers and five repeated assessments in each hand. These measures test strength and integration of motor and sensory feedback pathways.

If pinch or grip strength have decreased ( $>1$ SD on five repeated measurements compared to baseline), the treatment will be stopped and the child will receive intensive bimanual strength and sensory therapy with continuous monitoring until there is no difference in measurements from baseline.

\section{ASSESSMENT METHODS}

\section{Overview of assessments and variables derived}

Each component of the intervention has a corresponding outcome assessment or construct (table 1). These constructs have been validated in the literature and performed in our laboratories. Enhancement of sensory feedback has as its outcome sensory processing; training of reach has kinematics as an outcome; and the constraint-use itself has the outcome of change in motor development. Additionally, severity of CP will be classified at baseline.

The primary outcomes measures will include changes in somatosensory processing as measured by the ERP paradigm, improvements in reaching ability as measured by kinematics of reach assessment, and improvements in fine motor development as measured by BSIDIII.

\section{Classification of severity of CP}

Rationale: Although asymmetry is distinguishable in very young children with CP, tone characteristics are still evolving (especially in preterm infants) making it extremely difficult to attribute classifications of spasticity, diskynesia or ataxia. $^{71}$ The degree of fine motor function impairment in hemiparetic or highly asymmetric children with $\mathrm{CP}$ can be classified using standardised systems such as the Bimanual Fine Motor Function system (BFMF) ${ }^{107}$ or the Manual Abilities Classification system (MACS). ${ }^{108}$ The Surveillance of Cerebral Palsy in Europe (SCPE) group recommendations for classification of CP were to use the BFMF as it 'takes into account possible asymmetry while MACS does not'. ${ }^{109}$ The BFMF also fits well with the WHO 'components of health' framework rather than the earlier models of 'consequences of disease', in which activity limitations vary widely depending on the setting. ${ }^{107}$

Scoring: The study therapists will classify severity of CP in every child using the BFMF at their baseline assessment. The BFMF will be used to quantify baseline CP severity and used in the analysis as described.

\section{Somatosensory processing measurement by ERP}

Recording: A high-density array of 128 electrodes embedded in soft sponges (Geodesic Sensor Net, EGI, Inc., Eugene, OR) will be used to record ERPs with a sampling rate of $1000 \mathrm{~Hz}$, filters set to $0.1-400 \mathrm{~Hz}$ (figure 6).

All electrodes will be referred to $\mathrm{Cz}$ and re-referenced offline to an average reference. Recording of brainwaves will be controlled by Net Station (v. 4.3; EGI, Inc., Eugene, Oregon, USA). E-Prime (v. 2.0, PST, Inc., Pittsburgh, Pennsylvania, USA) software will control stimulus delivery. Air puffs will be delivered using a custom-made hand mold apparatus (Facility for Arts Research, Florida State University, Tallahassee, Florida, USA) with two flexible nozzles, one positioned approximately $0.5^{\prime \prime}$ below the palm of hand secured in a molded holder (puff condition), the other placed in proximity but directed away (sham condition). The pressure at the skin surface is 5 psi, or less than the pressure of the smallest microfilament used to test for neuropathies. ${ }^{54}$ For each hand, 60 puffs will be presented randomly interspersed with 60 sham trials. To prevent habituation, no more than two repetitions occur in a row, and intertrial intervals vary randomly between 2000 and $2500 \mathrm{~ms}$. The entire test session will last 8-10 min.

Table 1 Constructs and procedures

\begin{tabular}{|c|c|c|c|}
\hline Construct & Procedure(s) & $\begin{array}{l}\text { Duration } \\
\text { (min) }\end{array}$ & Variable \\
\hline Severity of $\mathrm{CP}$ & $\begin{array}{l}\text { Bimanual Fine Motor } \\
\text { Function scale }\end{array}$ & 5 & $\begin{array}{l}\text { Categories for fine motor impairment reflecting uni and } \\
\text { bimanual impairments }\end{array}$ \\
\hline $\begin{array}{l}\text { Somatosensory } \\
\text { processing }\end{array}$ & ERP to air puff & 10 & $\begin{array}{l}\text { Average amplitudes in } \mathrm{mV} \text { across predefined, published time } \\
\text { windows after stimulus onset, centroparietal and central } \\
\text { electrode clusters }\end{array}$ \\
\hline Reaching ability & Kinematics of reach & 15 & $\begin{array}{l}\text { Sum number of movement units (smoothness), time from } \\
\text { presentation to first contact, time from first contact to grasp, } \\
\text { approach velocity, scores using a motion-tracking system }\end{array}$ \\
\hline $\begin{array}{l}\text { Fine motor } \\
\text { development }\end{array}$ & $\begin{array}{l}\text { BSIDIII fine/gross } \\
\text { motor domains }\end{array}$ & 15 & $\begin{array}{l}\text { Composite standardised scorer for bimanual function, scaled } \\
\text { scores for unimanual function, normed for CA }\end{array}$ \\
\hline
\end{tabular}



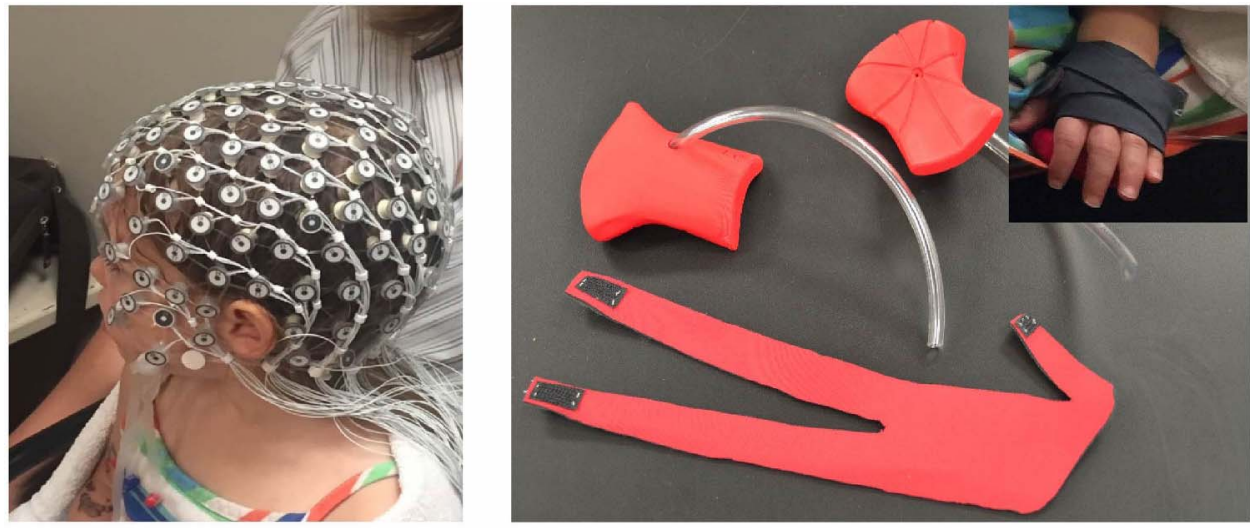

Image provided by the Facility for Arts Research (FAR)

Figure 6 Somatosensory ERP: geodesic ERP net and Air puff stimulus. ERP, event-related potential.

To facilitate cooperation, children will be offered an age-appropriate video.

\section{Kinematics of reach}

Reaching is a developmentally relevant task representative of a skilled movement. In children with CP, reach is impaired in its smoothness, range and accuracy. These qualities can be quantified using kinematics or the study of shape of movement path, velocity and acceleration. Kinematics of reach are well studied and validated both in TD and children with CP and allow an objective evaluation of performance.

Procedure: The child seated in a supportive chair (with trunk strap support if needed) will be asked to reach unassisted for an object placed on a prepositioned shelf at midline. The task will be repeated for three trials in three conditions: reach with the less affected arm (condition 1) and with the more affected arm (condition 2), then reach for a larger object with both arms (condition 3) to evaluate spontaneous and bilateral reach.

Recording: We will use a motion analysis system (Vicon Motion Systems Ltd, UK) including 4 infrared 'Bonita 3' cameras that track reflective markers at a rate of $240 \mathrm{~Hz}$ with spatial precision at the $1 \mathrm{~mm}$ level to capture the child's movement in three-dimensional space. Markers will be positioned bilaterally on the head of the second metacarpal bone and on the object. ${ }^{110-113}$ Vicon Tracker software will be used to determine the three-dimensional position of the hand as it approaches each object, and to construct segments, angles and moments using Matlab software (The MathWorks Inc., Natick, Massachusetts, USA).

Scoring: For each trial, approach velocity and movement units (marked by changes in speed or position) will be calculated. A single movement unit is defined as the portion of reach between one acceleration and another. ${ }^{114}$ The sum composite of movement units in one reach attempt is defined as smoothness. ${ }^{115} 116$ The motion tracking data will also be used to determine the time from presentation to first contact, time from first contact to grasp and approach velocity on the unilateral task. In addition to these measures, in the bilateral task, spontaneous use of the more versus less affected extremity will be scored.

\section{Bayley Scales of Infant and Toddler Development (Bayley III) - 3rd Edition}

Rationale: The Bayley III is the gold standard for the evaluation of former NICU graduates, especially preterm infants, and we have extensive experience using it in the follow-up clinic. ${ }^{75} 45{ }^{46}$ The motor scales provide a developmental and functional assessment, address unimanual as well as bimanual skills, are normed against a large population and designed to be adjusted for varying degrees of prematurity.

Procedure: The Bayley fine and gross motor subscales are currently administered in the follow-up clinic by trained examiners who undergo yearly retraining to maintain standards. The Bayley fine motor items for 6-24 months (score range: 0-42) measure prehension, motor planning, grasping patterns, and eye-hand coordination. The examiner is masked to intervention versus control group membership and administers the fine motor subscale twice, repeating unilateral items for right and left hands. Most (about 80\%) of the Bayley fine motor items for infants aged 6-24 months require unilateral reach and grasp.

Scoring: In addition to standard testing, which gives a composite score of the severity of motor delay or impairment in young children and is a bimanual estimate, separate scores will be obtained to characterise the difference between more and less affected extremity. Using the method of Lowes et al, ${ }^{117}$ after standard testing, unilateral items are repeated with the affected extremity, using a soft sling constraint if needed (as in the reach assessment). Scores for the unilateral items are added to the bilateral items to produce a raw score for each hand. ${ }^{117}$ All scores are corrected for GA. 
Feasibility of assessments

We have conducted non-invasive ERP and neurobehavioral studies in NICU infants and in older children (see preliminary data) and can reliably test up to 10 children 5-12 years old using four separate assessments (total 100 minutes testing) in a single day. In the past 3 years, our loss to follow-up at 6 months was less than $10 \%$. Missing data rates for all testing sessions were less than $5 \%$. This high rate of successful data collection is due to all children being tested on a single day to accommodate parent work-schedules and child care-schedules, as well as a streamlined testing design during which children rotate through research stations in scheduled time blocks. Total assessment time in the current proposal design is less than $1 \mathrm{~h}$ with a 5 min break between all stations, and appropriate play and snack areas in the testing suite, making testing tolerable for very young children and their parents.

\section{ANALYSIS PLAN}

Our first aim is to demonstrate that CIMT improves the sensory and motor function of an affected upper extremity in young children with asymmetric CP. We will use separate linear regression models to determine if, at 1-month and 7-month time points, ERP mean amplitudes, reach precision, and Bayley motor scores differ by intervention group in our CP participants. Each regression model will include an indicator variable for intervention status and adjust for baseline CP severity to increase the precision of the model. There are multiple clinical end points in this study. We wish to answer separate questions and will report all negative as well as positive results, so no multiplicity corrections will be made for the number of end points. ${ }^{118}$ The robust sandwich estimator of the variance will be used to relax the constant variance assumption in all regression models. Secondary models will also adjust for lesion pattern on neuroimaging $^{118}$ and more affected side. Additionally, secondary analyses will determine if duration of CIMT intervention (Fitbit data) is associated with each outcome. In the regression model, the indicator variable for intervention status will be replaced with a continuous function of the duration (average hours per day) of CIMT wear. Duration will be flexibly modelled using restricted cubic splines to describe any non-straight-line association and suggest a minimal duration needed to see an effect of CIMT.

Sample power: For this aim, we are studying only the 36 participants with CP, randomised to receive CIMT immediately (intervention group), and the 36 participants with CP in the control group, not receiving CIMT during the first 7 months of study involvement. We will use linear regression to estimate treatment effect while adjusting for baseline CP severity as measured using the BFMF. Including an adjustment variable in the model will allow us to detect smaller effect sizes with the same number of participants. The magnitude of the
Table 2 Percentage of variability by baseline CP severity

\begin{tabular}{llllll}
\hline$R_{y, z}^{2}$ & 0 & 0.05 & 0.10 & 0.20 & 0.30 \\
Std Effect Size & 0.78 & 0.74 & 0.70 & 0.62 & 0.54 \\
\hline
\end{tabular}

improvement will depend on the percentage of variability in each outcome that is explained by baseline CP severity $\left(\mathrm{R}_{\mathrm{Y}, \mathrm{Z}}^{2}\right)$ as given in table 2 .

If baseline CP severity and the outcome are uncorrelated, we will have $90 \%$ power to detect a 0.78 SD difference between intervention groups; if $\mathrm{R}_{\mathrm{Y}, \mathrm{Z}}^{2}=0.30$, we will have $90 \%$ power to detect a 0.54 SD difference between intervention groups. Our hypothesis is that CIMT will result in acute increases of absolute ERP mean amplitudes (detection and attention to stimulus), and in even greater effects by 3 months. We do not anticipate 1 month changes in bimanual motor function on the Bayley III, but do expect to see improvements in standardised scores for at 7 months as referenced to the unaffected extremity. We anticipate 1 month improvement in the more-affected extremity function on the Bayley III and equal or greater effects at the 7-month assessment. Improvements in reach kinematics and in more-affected extremity performance on the Bayley III should be tightly correlated to changes in ERP variables.

\section{Our second aim is to characterise the association of cortical sensory processing and motor function in the upper extremities of young children with asymmetric CP before CIMT}

The primary goal of aim 2 is to quantify the differences in sensory responses (mean amplitudes on ERP) and motor function (reach kinematics, Bayley-scaled scores) between (1) affected extremities of children with CP and (2) those of upper extremities in age-matched children without CP. A comparison between more-affected and less-affected sides in children with $\mathrm{CP}$ will also allow us to have a within-participants measure of asymmetry. Increases in unimanual function should also result in increasing bimanual function on the Bayley III.

The secondary goal of the analysis will correlate ERP mean amplitudes of predefined peaks with measures of motor function in children with CP. We hypothesise that improved cortical sensory processing will predict increased unimanual and bimanual upper extremity motor function.

Sample power: We are planning a study with $72 \mathrm{CP}$ and the set of $72 \mathrm{TD}$ participants matched at the baseline assessment. In a previous study, we found that the SD of ERP amplitudes was $1.8 \mu \mathrm{V}$ at P50, 3.6 $\mu \mathrm{V}$ at N140, and $3.2 \mu \mathrm{V}$ at P200. We will be able to detect a true difference in mean amplitude between $\mathrm{CP}$ and TD participants of $0.98 \mu \mathrm{V}(\mathrm{P} 50), 2.0 \mu \mathrm{V}(\mathrm{N} 140)$, and $1.7 \mu \mathrm{V}$ (P200) with power $0.90(\alpha=0.05)$. In the pilot data, we saw mean peak differences that were between 0.39 and 2.48 units between affected and less-affected extremity. Therefore, we are using a very conservative estimate of 
difference for our calculation: the effect size of between-TD-versus-CP differences is expected to be greater than that assumed for the affected versus less-affected variables in children with CP. For other outcomes, we have $90 \%$ power to detect a 0.54 SD difference between $\mathrm{CP}$ and TD, a small difference given published motor function differences $>2$ SD. For aim 2B, we will have $90 \%$ power to detect a correlation of 0.37 between ERP, Bayley, and reach kinematics in the 72 CP participants. ERPs in participants with CP should show decreased detection and awareness of light touch as measured by absolute mean amplitudes in affected extremities versus less-affected and versus TD children. Decreased cortical processing should in turn correlate with increased smoothness of reach, decreased approach velocity and higher Bayley III composite and affected extremity scores. Based on sensorimotor principles of learning, children with CP with worse sensory function should have decreased performance on reach kinematics and Bayley III as compared to those with sensory function closer to that of TD children.

\section{The third aim is to characterise the change of sensory and motor function of children with CP receiving CIMT as referenced to TD children}

Rationale: Published motor function trajectories in CP indicate that gross motor function throughout childhood improves but does not reach levels comparable to TD children. ${ }^{119}$ The gains achieved and the maintenance of gains are dependent on the severity of disease at baseline, with more severely affected children having more limited trajectories and even losing childhood gains into adulthood. Upper extremity function is less well studied, but similar patterns have been demonstrated in the more-affected extremity. ${ }^{120} 121$ Children receiving standard clinical therapies show few improvements over the course of 12 months,${ }^{24}$ as measured by kinematics or neurobehavioral measures. The function of the more-affected extremity can decline until 18 years of age, even as the less-affected extremity continues to improve. This disparate combination of trajectories of the more-affected and less-affected extremities results in patterns of bimanual function that do not reach TD levels. ${ }^{122}$

The main goal of the third aim is to use TD referenced-measures of function in children with $\mathrm{CP}$ who received CIMT to examine change from baseline to 6 months after intervention as correlated with CP severity at start of intervention.

Another goal is to examine the association of CA at CIMT on trajectories of sensory and motor function in children with CP as referenced to TD children.

Sample power: Sample size estimates are typically based on assuming a relevant effect size to detect with a given power. However, for aim 3, the research goal is to show that CP participants are becoming more similar to TD participants over time by showing the standardised units getting closer to zero. As discussed by others, ${ }^{123}$ in these instances, the sample size can be justified in terms of the margin of error (half the $95 \%$ CI width) of our estimate. For the purposes of the power calculation, we assume that the standardised units will have a variance of approximately 1 . With $72 \mathrm{CP}$ participants, we will be able to estimate the mean at study end with a margin of error of 0.24 standardisation units. When testing for difference by timing of CIMT intervention, the margin of error will be no larger than 0.33 standardisation units; as in aim 2, the margin of error will decrease as the correlation between baseline and study-end-standardised units increases. In contrast to findings in children with $\mathrm{CP}$ described above, we hypothesise that as DD improves after CIMT, we will observe increases in performance metrics on all cortical and neurobehavioral assessments in the more-affected extremity as well as in bimanual function, as referenced to TD children. If the BFMF, which includes five levels, does not provide sufficient precision for modelling of impairment, we will use the composite Bayley III motor score, acknowledging that it is more a direct measure of ability rather than impairment.

\section{Data management}

Only the Principal Investigator and the research coordinator will have access to individually identifiable private information about human participants.

A Data Safety Monitoring Committee (DSMC) will include the PI, two additional physicians, and an independent community occupational therapist. The DSMC will meet every 6 months to discuss the project and will receive enrolment, adverse events and compliance reports. The DSMC will have access to interim results and make the final decision to terminate the trial.

Data and Safety reports: Safety reports will include any problems with compliance, adverse events, withdrawals from the study, reports and events submitted to the IRB. Data reports will include enrolment in all groups and timelines. Adverse events will be evaluated on a continuous basis with review by the DSMC. After 36 CP patients have been enrolled (halfway point) an interim analysis of upper extremity function effects only will be performed and provided to the DSMC. Because the intervention has been shown to be beneficial with no major or lasting concerns and the wait-list controls will also receive the intervention, a limited interim analysis will not jeopardise the final results.

Any data on medical information and behavioural assessments will be directly collected into the Research Electronic Data Capture (RedCap), relying on a thorough study-specific data dictionary defined in an iterative self-documenting process by all members of the research team with planning assistance from the RIC.

We hope to refine the CIMT intervention for future proposals and for clinical practice. Associations between sensory function at baseline and improvements in sensory processing could prove invaluable to the design of further training tasks to maximise sensory feedback during movement. Associations between severity of CP and effectiveness of treatment may help decide which 
children will benefit most from CIMT versus other standard therapies. Additionally, using quality improvement techniques in single centres or collaboratives, the intervention could be implemented in non-academic settings such as rural areas or resource-poor countries, with the same CIMT design but less resource-intensive end points, such as questionnaires.

\section{Author affiliations}

${ }^{1}$ The Perinatal Research Institute at Nationwide Children's Hospital, Columbus, Ohio, USA

${ }^{2}$ Department of Allied Health and Rehabilitation Sciences, The Ohio State University, Columbus, Ohio, USA

${ }^{3}$ Department of Hearing and Speech Sciences, Vanderbilt University Medical Center, Nashville, Tennessee, USA

${ }^{4}$ Department of Pediatrics, Nationwide Children's Hospital, Columbus, Ohio, USA

${ }^{5}$ Department of Clinical Neurosciences and Department of Radiology, Centre Hospitalier Universitaire Vaudois, Lausanne, Switzerland

${ }^{6}$ Department of Psychology, Vanderbilt University, Nashville, Tennessee, USA ${ }^{7}$ Department of Biostatistics, Vanderbilt University Medical Center, Nashville, Tennessee, USA

Contributors OC helped draft the initial manuscript, designed the process flows for the study, reviewed the manuscript and approved the final protocol manuscript as submitted. JH participated in the design of the biomechanics and kinematics assessments and the reach training, revised the manuscript and approved the final protocol manuscript as submitted. AK participated in the design of the electrophysiology assessments, revised the manuscript and approved the final protocol manuscript as submitted. GN HC and MAN participated in the design of the cerebral palsy assessments, revised the manuscript and approved the final protocol manuscript as submitted. EH helped design the process flows for the study, reviewed the manuscript and approved the final protocol manuscript as submitted. MM conceptualised the design of the electrophysiology analysis, revised the manuscript and approved the final protocol manuscript as submitted. AN conceptualised the design of the 'Sticky Mittens' and participated in the design of the reach training, revised the manuscript and approved the final protocol manuscript as submitted. JCS conceptualised the statistical analysis approach with NLM, helped draft the initial manuscript, reviewed the manuscript and approved the final protocol manuscript as submitted. NLM conceptualised and designed the study, obtained funding for the study, selected the data collection instruments, coordinated the plan for the initial and advanced statistical analyses, helped draft the initial manuscript and all drafts, and approved the final protocol manuscript as submitted.

Funding This work is supported by National Institutes of Child Health and Human Development, grant number [R01HD081120-01A1].

Competing interests None declared.

Patient consent Obtained.

Ethics approval Nationwide Children's Hospital Institutional Review Board, IRB \#15-00547.

Provenance and peer review Not commissioned; peer reviewed for ethical and funding approval prior to submission.

Open Access This is an Open Access article distributed in accordance with the Creative Commons Attribution Non Commercial (CC BY-NC 4.0) license, which permits others to distribute, remix, adapt, build upon this work noncommercially, and license their derivative works on different terms, provided the original work is properly cited and the use is non-commercial. See: http:// creativecommons.org/licenses/by-nc/4.0/

\section{REFERENCES}

1. Reddihough DS, Collins KJ. The epidemiology and causes of cerebral palsy. Aust J Physiother 2003;49:7-12.
2. Yeargin-Allsopp M, Van Naarden Braun K, Doernberg NS, et al. Prevalence of cerebral palsy in 8-year-old children in three areas of the United States in 2002: a multisite collaboration. Pediatrics 2008;121:547-54.

3. Bhasin TK, Brocksen S, Avchen RN, et al. Prevalence of four developmental disabilities among children aged 8 yearsMetropolitan Atlanta Developmental Disabilities Surveillance Program, 1996 and 2000. MMWR Surveill Summ 2006;55:1-9.

4. Cruz M, Jenkins R, Silberberg $D$. The burden of brain disorders. Science (New York, NY) 2006; 312:53-53.

5. Tachdjian MO, Minear WI. Sensory disturbances in the hands of children with cerebral palsy. J Bone Joint Surg Am 1958:40-A:85-90.

6. Yekutiel M, Jariwala M, Stretch P. Sensory deficit in the hands of children with cerebral palsy: a new look at assessment and prevalence. Dev Med Child Neurol 1994;36:619-24.

7. O'Shea TM. Cerebral palsy in very preterm infants: new epidemiological insights. Ment Retard Dev Disabil Res Rev 2002;8:135-45

8. Rosenbaum $\mathrm{P}$, Paneth $\mathrm{N}$, Leviton $\mathrm{A}$, et al. A report: the definition and classification of cerebral palsy. April 2006. Dev Med Child Neurol Suppl 2007;109:8-14.

9. Rosenbaum P, Stewart D. The world health organization international classification of functioning, disability, and health a model to guide clinical thinking, practice and research in the field of cerebral palsy. Seminars Pediatr Neurol 2004;11:5-10.

10. Strauss D, Brooks J, Rosenbloom L, et al. Life expectancy in cerebral palsy: an update. Dev Med Child Neurol 2008;50:487-93.

11. Westbom L, Bergstrand L, Wagner $P$, et al. Survival at 19 years of age in a total population of children and young people with cerebral palsy. Dev Med Child Neurol 2011;53:808-14.

12. Brooks J, Day S, Shavelle R, et al. Low Weight, Morbidity, and Mortality in Children With Cerebral Palsy: New Clinical Growth Charts. Pediatrics 2011:128:e299-307.

13. Hutton JL, Pharoah PO. Effects of cognitive, motor, and sensory disabilities on survival in cerebral palsy. Arch Dis Child 2002;86:84-9.

14. Hutton JL, Pharoah PO. Life expectancy in severe cerebral palsy. Arch Dis Child 2006;91:254-8.

15. Kancherla V, Amendah DD, Grosse SD, et al. Medical expenditures attributable to cerebral palsy and intellectual disability among Medicaid-enrolled children. Res Dev Disabil 2012;33:832-40.

16. Kruse M, Michelsen SI, Flachs EM, et al. Lifetime costs of cerebral palsy. Dev Med Child Neurol 2009;51:622-8.

17. Honeycutt AA, Grosse SD, Dunlap LJ, et al. Economic costs of mental retardation, cerebral palsy, hearing loss, and vision impairment. In: Altman BM, Barnartt SN, Hendershot G, Larson SA eds. Using Survey Data to Study Disability, Volume 3: Results from the National Health Survey on Disability. Kidlington, Oxford, UK: Elsevier / JAI Press 2003:207-28.

18. Novak I, McIntyre S, Morgan C, et al. A systematic review of interventions for children with cerebral palsy: state of the evidence. Dev Med Child Neurol 2013:55:885-910.

19. Eliasson AC, Gordon AM, Forssberg H. Impaired anticipatory control of isometric forces during grasping by children with cerebral palsy. Dev Med Child Neurol 1992;34:216-25.

20. Auld ML, Boyd RN, Moseley GL, et al. Impact of tactile dysfunction on upper-limb motor performance in children with unilateral cerebral palsy. Arch Phys Med Rehabil 2012;93:696-702.

21. DeLuca SC, Echols K, Ramey SL, et al. Pediatric constraint-induced movement therapy for a young child with cerebral palsy: two episodes of care. Phys Ther 2003;83:1003-13.

22. Taub E, Ramey SL, DeLuca S, et al. Efficacy of constraint-induced movement therapy for children with cerebral palsy with asymmetric motor impairment. Pediatrics 2004;113:305-12.

23. Houwink A, Aarts PB, Geurts AC, et al. A neurocognitive perspective on developmental disregard in children with hemiplegic cerebral palsy. Res Dev Disabil 2011;32:2157-63.

24. Hanna SE, Law MC, Rosenbaum PL, et al. Development of hand function among children with cerebral palsy: growth curve analysis for ages 16 to 70 months. Dev Med Child Neurol 2003; 45:448-55.

25. Hanna SE, Rosenbaum PL, Bartlett DJ, et al. Stability and decline in gross motor function among children and youth with cerebral palsy aged 2 to 21 years. Dev Med Child Neurol 2009;51:295-302.

26. Vos RC, Becher JG, Ketelaar M, et al. Developmental trajectories of daily activities in children and adolescents with cerebral palsy. Pediatrics 2013;132:e915-23.

27. Beckung E, Carlsson G, Carlsdotter S, et al. The natural history of gross motor development in children with cerebral palsy aged 1 to 15 years. Dev Med Child Neurol 2007;49:751-6. 
28. Beckung E, Hagberg G, Uldall P, et al. Surveillance of Cerebral Palsy in Europe. Probability of Walking in Children With Cerebral Palsy in Europe. Pediatrics 2008;121:e187-92.

29. Bult MK, Verschuren O, Jongmans MJ, et al. What influences participation in leisure activities of children and youth with physical disabilities? A systematic review. Res Dev Disabil 2011;32:1521-9.

30. Öhrvall AM, Eliasson AC, LÖWING K, et al. Self-care and mobility skills in children with cerebral palsy, related to their manual ability and gross motor function classifications. Dev Med Child Neurol 2010;52:1048-55

31. Smits DW, Ketelaar M, Gorter JW, et al. Development of daily activities in school-age children with cerebral palsy. Res Dev Disabil 2011;32:222-34.

32. van Eck M, Dallmeijer AJ, van Lith IS, et al. Manual ability and its relationship with daily activities in adolescents with cerebral palsy. $J$ Rehabil Med 2010;42:493-8.

33. Chouinard P, Leonard G, Paus T. Changes in effective connectivity of the primary motor cortex in stroke patients after rehabilitative therapy. Exp Neurol 2006;201:375-87.

34. Sakzewski L, Carlon S, Shields N, et al. Impact of intensive upper limb rehabilitation on quality of life: a randomized trial in children with unilateral cerebral palsy. Dev Med Child Neurol 2012:54:415-23.

35. Hoare BJ, Wasiak J, Imms C, et al. Constraint-induced movement therapy in the treatment of the upper limb in children with hemiplegic cerebral palsy. Cochrane Database Syst Rev 2007;(2): CD004149.

36. Hoare B, Imms C, Carey L, et al. Constraint-induced movement therapy in the treatment of the upper limb in children with hemiplegic cerebral palsy: a Cochrane systematic review. Clin Rehabil 2007;21:675-85.

37. Johnston MV. Clinical disorders of brain plasticity. Brain Dev 2004;26:73-80.

38. Johnston MV. Injury and plasticity in the developing brain. Exp Neurol 2003;184(Suppl 1):S37-41.

39. Charles J, Gordon AM. A critical review of constraint-induced movement therapy and forced use in children with hemiplegia Neural Plast 2005;12:245-61; discussion263-72.

40. Charles JR, Gordon AM. A repeated course of constraint-induced movement therapy results in further improvement. Dev Med Child Neurol 2007;49:770-3.

41. Charles JR, Wolf SL, Schneider JA, et al. Efficacy of a child-friendly form of constraint-induced movement therapy in hemiplegic cerebral palsy: a randomized control trial. Dev Med Child Neurol 2006;48:635-42.

42. Taub E, Griffin A, Uswatte G, et al. Treatment of congenital hemiparesis with pediatric constraint-induced movement therapy. J Child Neurol 2011;26:1163-73.

43. Deluca SC, Echols K, Law CR, et al. Intensive pediatric constraint-induced therapy for children with cerebral palsy: randomized, controlled, crossover trial. J Child Neurol 2006;21:931-8.

44. Parkes J, Donnelly C, McDowell B, et al. Recruitment bias and characteristics of participants with severe cerebral palsy in a cross-sectional survey. J Adv Nurs 2012;68:368-78.

45. Palisano RJ, Almarsi N, Chiarello LA, et al. Family needs of parents of children and youth with cerebral palsy. Child Care Health Dev 2010;36:85-92.

46. Schuengel C, Rentinck IC, Stolk J, et al. Parents' reactions to the diagnosis of cerebral palsy: associations between resolution, age and severity of disability. Child Care Health Dev 2009;35:673-80.

47. Johnston MV, Ishida A, Ishida WN, et al. Plasticity and injury in the developing brain. Brain Dev 2009;31:1-10.

48. Johnston MV. Plasticity in the developing brain: implications for rehabilitation. Dev Disabil Res Rev 2009;15:94-101.

49. Ramey SL. Multisite RCT of 3 neurorehabilitation therapies for infants with asymmetrical. NIH Research Portfolio Online Reporting Tools, 2014. https://projectreporter.nih.gov

50. Maitre NL, Aschner JL, Slaughter JC, et al. Cortical speech sound differentiation in the intensive care nursery predicts cognitive and language development in the first 2 years of life. Dev Med Child Neurol 2013;55:834-9.

51. Maitre NL, Slaughter JC, Aschner JL. Early prediction of cerebral palsy after neonatal intensive care using motor development trajectories in infancy. Early Human Development. Elsevier, 2013.

52. Maitre NL, Smolinsky C, Slaughter JC, et al. Adverse neurodevelopmental outcomes after exposure to phenobarbital and levetiracetam for the treatment of neonatal seizures. J Perinato 2013;33:841-6.

53. Badawi N, Keogh JM. Causal pathways in cerebral palsy. $J$ Paediatr Child Health 2013;49:5-8.
54. Maitre NL, Barnett ZP, Key AP. Novel assessment of cortical response to somatosensory stimuli in children with hemiparetic cerebral palsy. J Child Neurol 2012;27:1276-83.

55. Karniski W. The late somatosensory evoked potential in premature and term infants. I. Principal component topography. Electroencephalogr Clin Neurophysiol 1992;84:32-43.

56. Sterling C, Taub E, Davis D, et al. Structural neuroplastic change after constraint-induced movement therapy in children with cerebral palsy. Pediatrics 2013;131:e1664-9.

57. Mark VW, Taub E, Morris DM. Neuroplasticity and constraint-induced movement therapy. Eura Medicophys 2006;42:269-84.

58. Boyd LA, Vidoni ED, Daly JJ. Answering the call: the influence of neuroimaging and electrophysiological evidence on rehabilitation. Phys Ther 2007;87:684-703.

59. Auld ML, Ware RS, Boyd RN, et al. Reproducibility of tactile assessments for children with unilateral cerebral palsy. Phys Occup Ther Pediatr 2012;32:151-66.

60. Auld M. Tactile function in the upper limb of children with unilateral cerebral palsy. Australia: University of Queensland, 2011.

61. Gauthier LV, Taub E, Perkins C, et al. Remodeling the brain: plastic structural brain changes produced by different motor therapies after stroke. Stroke 2008;39:1520-5.

62. Sampaio E, Philip J. Sensory and motor aspects of pseudoneglect, hemifield, and hemispace in the tactile modality. Brain Cogn 1991;16:74-82.

63. Taub E, Uswatt G. Constraint-Induced Movement therapy: answers and questions after two decades of research. NeuroRehabilitation 2006;21:93-5.

64. Taub E. Harnessing brain plasticity through behavioral techniques to produce new treatments in neurorehabilitation. Am Psychol 2004:59:692-704.

65. Houwink A, Geerdink YA, Steenbergen B, et al. Assessment of upper-limb capacity, performance, and developmental disregard in children with cerebral palsy: validity and reliability of the revised Video-Observation Aarts and Aarts module: Determine Developmental Disregard (VOAA-DDD-R). Dev Med Child Neurol 2013;55:76-82.

66. Aarts $\mathrm{P}$, Jongerius $\mathrm{P}$, Geerdink $\mathrm{Y}$, et al. Validity and reliability of the VOAA-DDD to assess spontaneous hand use with a video observation tool in children with spastic unilateral cerebral palsy. BMC musculoskelet Disord 2009;10:145

67. McMillan JH. Randomized field trials and internal validity: Not so fas my friend. Pract Assess Res Eval 2007;12 2-13. ISSN 1531-7714.

68. West SG, Sagarin BJ. Participant selection and loss in randomized experiments. Research design: Donald Campbell's legacy 2 2000:117-154

69. Shadish W, Cook T, Campbell D. Experimental And QuasiExperimental Designs For Generalized Causal Inference. Boston: Houghton Mifflin; 2001.

70. Maitre NL, Wang L, Gogliotti S, et al. Constraint-Induced Movement Therapy Improves Cortical Sensory Perception and Upper Extremity Function in Children with Hemiparesis. Pediatric Academic Societies meeting. Washington, DC; 2013.

71. Engle WA, American Academy of Pediatrics Committee on Fetus and Newborn. Age Terminology During the Perinatal Period. Pediatrics 2004:114:1362-4

72. Kuban K, Allred E, O'shea M, et al. An algorithm for identifying and classifying cerebral palsy in young children. $J$ Pediatr 2008:153:466-472.e1.

73. Kuban KC, O'Shea M, Allred E, et al. Video and CD-ROM as a training tool for performing neurologic examinations of 1 -year-old children in a multicenter epidemiologic study. J Child Neurol 2005;20:829-31.

74. Palisano RJ, Rosenbaum $\mathrm{P}$, Bartlett $\mathrm{D}$, et al. Content validity of the expanded and revised Gross Motor Function Classification System. Dev Med Child Neurol 2008;50:744-50.

75. Albers CGA. Test review: Bayley scales of infant and toddler development, third edition. J Psychoeducational Assess 2007;25:180-90

76. Charles J, Lavinder G, Gordon AM. Effects of constraint-induced therapy on hand function in children with hemiplegic cerebral palsy. Pediatric Physical Therapy 2001;13:68-76.

77. Novak I, Cusick A, Lannin N. Occupational therapy home programs for cerebral palsy: double-blind, randomized, controlled trial. Pediatrics 2009;124:e606-14.

78. Hoon AH, Stashinko EE, Nagae LM, et al. Sensory and motor deficits in children with cerebral palsy born preterm correlate with diffusion tensor imaging abnormalities in thalamocortical pathways. Dev Med Child Neurol 2009;51:697-704.

79. Nagae LM, Hoon AH, Stashinko E, et al. Diffusion tensor imaging in children with periventricular leukomalacia: variability of injuries to white matter tracts. AJNR Am J Neuroradiol 2007;28:1213-22. 
80. Yoshida S, Hayakawa K, Yamamoto A, et al. Quantitative diffusion tensor tractography of the motor and sensory tract in children with cerebral palsy. Dev Med Child Neurol 2010;52:935-40.

81. Scheck SM, Boyd RN, Rose SE. New insights into the pathology of white matter tracts in cerebral palsy from diffusion magnetic resonance imaging: a systematic review. Dev Med Child Neurol 2012;54:684-96.

82. Williamson G, Anzalone M. Sensory Integration And SelfRegulation In Infants And Toddlers. Washington, DC: Zero to Three; 2001.

83. Kandel E, Schwartz J, Jessell T, et al. Principles of Neural Science. 5th edn. McGraw Hill Professional, 2012. ISBN-10: 0071390111.

84. Franklin DW, Wolpert DM. Computational mechanisms of sensorimotor control. Neuron 2011;72:425-42.

85. Wolpert DM, Diedrichsen J, Flanagan JR. Principles of sensorimotor learning. Nat Rev Neurosci 2011;12:739-51.

86. Körding KP, Wolpert DM. Bayesian decision theory in sensorimotor control. Trends Cogn Sci 2006;10:319-26.

87. Robert MT, Guberek R, Sveistrup H, et al. Motor learning in children with hemiplegic cerebral palsy and the role of sensation in short-term motor training of goal-directed reaching. Dev Med Child Neurol 2013;55:1121-8.

88. Bleyenheuft Y, Gordon AM. Precision grip control, sensory impairments and their interactions in children with hemiplegic cerebral palsy: a systematic review. Res Dev Disabil 2013;34:3014-28.

89. Bleyenheuft $\mathrm{Y}$, Thonnard JL. Predictive and reactive control of precision grip in children with congenital hemiplegia. Neurorehabil Neural Repair 2010;24:318-27.

90. Juenger $\mathrm{H}$, Linder-Lucht $\mathrm{M}$, Walther $\mathrm{M}$, et al. Cortical neuromodulation by constraint-induced movement therapy in congenital hemiparesis: an FMRI study. Neuropediatrics 2007;38:130-6.

91. Juenger $\mathrm{H}$, Kuhnke $\mathrm{N}$, Braun $\mathrm{C}$, et al. Two types of exercise-induced neuroplasticity in congenital hemiparesis: a transcranial magnetic stimulation, functional MRI, and magnetoencephalography study. Dev Med Child Neurol 2013;55:941-51.

92. Friel KM, Martin JH. Role of sensory-motor cortex activity in postnatal development of corticospinal axon terminals in the cat. J Comp Neurol 2005;485:43-56.

93. Friel K, Chakrabarty $\mathrm{S}, \mathrm{Kuo} \mathrm{HC}$, et al. Using motor behavior during an early critical period to restore skilled limb movement after damage to the corticospinal system during development. $J$ Neurosci 2012;32:9265-76.

94. Cooper J, Majnemer A, Rosenblatt B, et al. The determination of sensory deficits in children with hemiplegic cerebral palsy. J Child Neurol 1995;10:300-9.

95. Friel KM, Kuo HC, Carmel JB, et al. Improvements in hand function after intensive bimanual training are not associated with corticospinal tract dysgenesis in children with unilateral cerebral palsy. Exp Brain Res 2014;232:2001-9.

96. Gordon AM, Hung YC, Brandao M, et al. Bimanual training and constraint-induced movement therapy in children with hemiplegic cerebral palsy: a randomized trial. Neurorehabil Neural Repair 2011;25:692-702.

97. Aarts PB, Jongerius PH, Geerdink YA, et al. Effectiveness of modified constraint-induced movement therapy in children with unilateral spastic cerebral palsy: a randomized controlled trial. Neurorehabil Neural Repair 2010;24:509-18.

98. Aarts PB, Jongerius PH, Geerdink YA, et al. Modified Constraint-Induced Movement Therapy combined with Bimanual Training (mCIMT-BiT) in children with unilateral spastic cerebral palsy: how are improvements in arm-hand use established? Res Dev Disabil 2011;32:271-9.

99. Eliasson AC, Sjöstrand L, Ek L, et al. Efficacy of baby-CIMT: study protocol for a randomised controlled trial on infants below age 12 months, with clinical signs of unilateral CP. BMC Pediatr 2014;14:1-11.

100. Corbetta D, Snapp-Childs W. Seeing and touching: The role of sensory-motor experience on the development of infant reaching Infant Behav Dev 2009;32:44-58.

101. Maitre NL, Henderson G, Gogliotti S, et al. Feasibility of event-related potential methodology to evaluate changes in cortical processing after rehabilitation in children with cerebral palsy: A pilot study. J Clin Exp Neurophysiol 2014;36:669-79.
102. Needham A, Barrett T, Peterman K. A pick-me-up for infants' exploratory skills: Early simulated experiences reaching for objects using "sticky mittens" enhances young infants' object exploration skills. Infant Behav Dev 2002;25:279-95.

103. Libertus K, Needham A. Reaching experience increases face preference in 3-month-old infants. Developmental Sci 2011;14:1355-64.

104. Sommerville JA, Woodward AL, Needham A. Action experience alters 3-month-old infants" perception of others" actions. Cognition 2005;96:B1-11.

105. Woodward A, Needham A. Learning And The Infant Mind. Oxford : Oxford University Press, 2009.

106. Greaves S, Imms C, Krumlinde-Sundholm L, et al. Bimanual behaviours in children aged 8-18 months: A literature review to select toys that elicit the use of two hands. Res Dev Disabil 2012;33:240-50.

107. Beckung E, Hagberg G. Neuroimpairments, activity limitations, and participation restrictions in children with cerebral palsy. Dev Med Child Neurol 2002;44:309-16.

108. Eliasson AC, Krumlinde-Sundholm L, Rösblad B, et al. The Manual Ability Classification System (MACS) for children with cerebral palsy: scale development and evidence of validity and reliability. Dev Med Child Neurol 2006;48:549-54

109. Cans C, Dolk H, Platt MJ, et al, SCPE Collaborative Group. Recommendations from the SCPE collaborative group for defining and classifying cerebral palsy. Dev Med Child Neurol Suppl 2007;109:35-8.

110. Cimolin V, Beretta E, Piccinini L, et al. Constraint-induced movement therapy for children with hemiplegia after traumatic brain injury: a quantitative study. J Head Trauma Rehabil 2012;27:177-87.

111. Lin KC, Wu CY, Wei TH, et al. Effects of modified constraint-induced movement therapy on reach-to-grasp movements and functional performance after chronic stroke: a randomized controlled study. Clin Rehabil 2007;21:1075-86.

112. Bhat A, Heathcock J, Galloway JC. Toy-oriented changes in hand and joint kinematics during the emergence of purposeful reaching. Infant Behav Dev 2005;28:445-65.

113. Hung YC, Casertano L, Hillman A, et al. The effect of intensive bimanual training on coordination of the hands in children with congenital hemiplegia. Res Dev Disabil 2011;32:2724-31.

114. Kluzik J, Fetters L, Coryell J. Quantification of control: a preliminary study of effects of neurodevelopmental treatment on reaching in children with spastic cerebral palsy. Phys Ther 1990;70:65-76; discussion76-8.

115. Jaspers $\mathrm{E}$, Feys $\mathrm{H}$, Bruyninckx $\mathrm{H}$, et al. The reliability of upper limb kinematics in children with hemiplegic cerebral palsy. Gait Posture 2011;33:568-75

116. Rönnqvist $L$, Rösblad B. Kinematic analysis of unimanual reaching and grasping movements in children with hemiplegic cerebral palsy. Clin Biomech (Bristol, Avon) 2007;22:165-75.

117. Lowes LP, Mayhan M, Orr T, et al. Pilot Study of the Efficacy of Constraint-Induced Movement Therapy for Infants and Toddlers with Cerebral Palsy. Phys Occup Ther Pediatr 2014; 34:4-21.

118. Cook RJ, Farewell VT. Multiplicity Considerations in the Design and Analysis of Clinical Trials. J R Stat Soc Ser A Stat Soc 1996;159:93-110.

119. Rosenbaum PL, Walter SD, Hanna SE, et al. Prognosis for gross motor function in cerebral palsy: creation of motor development curves. JAMA 2002;288:1357-63.

120. Fedrizzi E, Pagliano E, Andreucci E, et al. Hand function in children with hemiplegic cerebral palsy: prospective followup and functional outcome in adolescence. Dev Med Child Neurol 2003;45:85-91.

121. Eliasson AC, Forssberg H, Hung YC, et al. Development of hand function and precision grip control in individuals with cerebral palsy: a 13-year follow-up study. Pediatrics 2006;118:e1226-36.

122. Holmefur M, Krumlinde-Sundholm L, Bergstrom J, et al. Longitudinal development of hand function in children with unilateral cerebral palsy. Dev Med Child Neurol 2010;52:352-7.

123. Parker RA, Berman NG. Sample Size. Am Statistician 2003;57:166-70. 